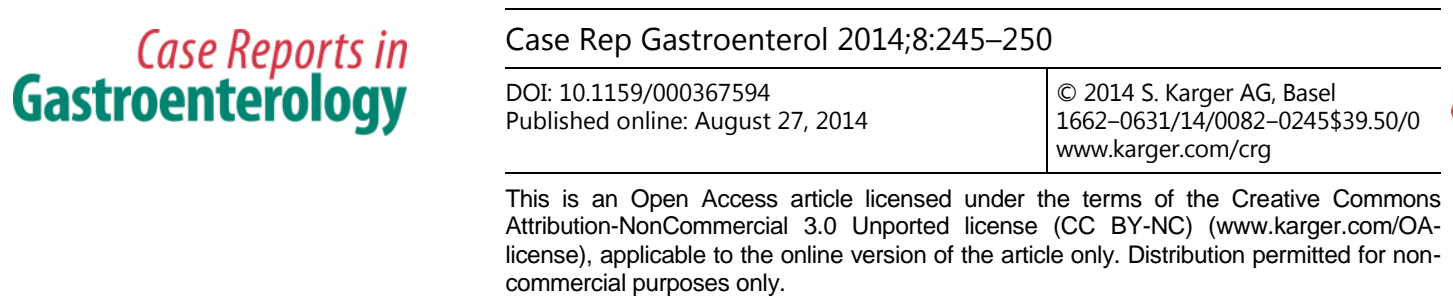

\title{
Endoscopic Sphincterotomy Using the Rendezvous Technique for Choledocholithiasis during Laparoscopic Cholecystectomy: A Case Report
}

\author{
Takayuki Tanaka $^{a}$ Masashi Haraguchi ${ }^{a}$ Hirotaka Tokai ${ }^{a}$ Shinichiro Ito ${ }^{a}$ \\ Masachika Kitajima $^{a}$ Tsuyoshi Ohno $^{a}$ Shinya Onizuka ${ }^{a} K_{\text {Keiji Inoue }}{ }^{a}$ \\ Yasuhide Motoyoshi $^{\text {b }}$ Tamotsu Kuroki $^{c}$ Takashi Kanemastu $^{\mathrm{a}}$ \\ Susumu Eguchic \\ Departments of a Surgery and ${ }^{b}$ Gastroenterology, Nagasaki Harbor Medical Center City \\ Hospital, and ' Department of Surgery, Nagasaki University Graduate School of Biomedical \\ Sciences, Nagasaki, Japan
}

\section{Key Words}

Endoscopic sphincterotomy · Laparoscopic cholecystectomy · Rendezvous technique

\begin{abstract}
A 50-year-old male was examined at another hospital for fever, general fatigue and slight abdominal pain. He was treated with antibiotics and observed. However, his symptoms did not lessen, and laboratory tests revealed liver dysfunction, jaundice and an increased inflammatory response. He was then admitted to our hospital and underwent an abdominal computed tomography scan and magnetic resonance cholangiopancreatography (MRCP), which revealed common bile duct (CBD) stones. He was diagnosed with mild acute cholangitis. As the same time, he was admitted to our hospital and an emergency endoscopic retrograde cholangiopancreatography was performed. Vater papilla opening in the third portion of the duodenum and presence of a peripapillary duodenal diverticulum made it difficult to perform cannulation of the CBD. In addition, MRCP revealed that the CBD was extremely narrow (diameter $5 \mathrm{~mm}$ ). We therefore performed laparoscopic cholecystectomy and endoscopic sphincterotomy using the rendezvous technique for choledocholithiasis simultaneously rather than laparoscopic CBD exploration. After the operation, the patient was discharged with no complications. Although the rendezvous technique has not been very commonly
\end{abstract}

Takayuki Tanaka

Department of Surgery, Nagasaki Harbor Medical Center City Hospital

6-391 Shinchi-machi, Nagasaki 850-8555 (Japan)

E-Mail pay-it-forward.197675@hotmail.co.jp 
Tanaka et al.: Endoscopic Sphincterotomy Using the Rendezvous Technique for Choledocholithiasis during Laparoscopic Cholecystectomy: A Case Report

used because several experts in the technique and a large operating room are required, this technique is a very attractive and effective approach for treating choledocholithiasis, for which endoscopic treatment is difficult.

(C) 2014 S. Karger AG, Basel

\section{Introduction}

There are three treatments for common bile duct (CBD) stones: (1) laparoscopic cholecystectomy (LC) after endoscopic treatment, in a two-step procedure, (2) performing cholecystectomy and CBD exploration simultaneously, and (3) performing cholecystectomy and CBD exploration simultaneously using a transcystic approach [1]. However, the standard treatment for CBD stones remains controversial. According to a questionnaire survey distributed by the Japan Society for Endoscopic Surgery in 2012 regarding the endoscopic treatment for choledocholithiasis, the use of a combination treatment of performing cholecystectomy (laparoscopic or open) after endoscopic treatment has been increasing year by year [2]. However, there are some cases in whom insertion of the cannula into the CBD during endoscopic retrograde cholangiopancreatography (ERCP) is difficult due to anatomical abnormalities, a duodenal diverticulum or other reasons [3]. We report the case of our patient, for whom we used the rendezvous technique during LC in an endoscopic sphincterotomy (EST).

\section{Case Report}

A 50-year-old male was examined at another hospital for fever, general fatigue and slight abdominal pain. He was treated with antibiotics and observed, but the next day, his symptoms were unchanged and he underwent laboratory tests, which revealed the following: WBC count 12,000 cells/ml, AST $300 \mathrm{IU} / \mathrm{l}$, ALT $500 \mathrm{IU} / \mathrm{l}$, ALP $550 \mathrm{IU} / \mathrm{l}$, and T-Bil $5.0 \mathrm{mg} / \mathrm{dl}$. He was then admitted to our hospital and underwent further laboratory tests, revealing a WBC count of 4,000 cells $/ \mathrm{ml}$ and a $\mathrm{Hb}$ level of $13.0 \mathrm{~g} / \mathrm{dl}$. Liver enzymes were as follows: AST 117 IU/l, ALT 295 IU/l, ALP 425 IU/l, T-Bil $1.8 \mathrm{mg} / \mathrm{dl}$, and CRP $4.22 \mathrm{mg} / \mathrm{dl}$. The diagnoses considered were cholecystitis and cholangitis.

Computed tomography and magnetic resonance cholangiopancreatography (MRCP) were then conducted. The computed tomography revealed a CBD stone and no findings suggestive of cholecystitis (fig. 1a). The MRCP showed a defect suggesting CBD stone; the CBD was extremely narrow (diameter $5 \mathrm{~mm}$ ) (fig. 1b). We diagnosed acute cholangitis due to a CBD stone.

Although an incarcerated bile duct stone may be released naturally, an emergency ERCP was performed in this case, but a peripapillary diverticulum made it impossible to insert the cannulation tube into the CBD (fig. 1c, d). A surgical procedure was thus performed as follows: (1) The patient was placed in the left semilateral position and was supine by being rotated $45^{\circ}$ to the right. A standard LC was then performed; the contrast medium was inserted via the cystic duct and the guide wire through the contrast tube was sent to the duodenum through the papilla of Vater from the CBD (fig. 2a, b). (2) For insertion of the guide wire, the patient was returned to the left semilateral position, the endoscope was inserted by the endoscopist, the guide wire was pulled out of the mouth, the cutting knife was inserted, and an intraoperative EST was performed (fig. 2c). (3) Stone removal with a balloon was performed (fig. 2d). The patient was returned to the supine position and the gallbladder was removed. He was discharged with no complications after the operation. 
Tanaka et al.: Endoscopic Sphincterotomy Using the Rendezvous Technique for Choledocholithiasis during Laparoscopic Cholecystectomy: A Case Report

\section{Discussion}

As is shown in the 2013 Tokyo Guidelines (in English) for the management of acute cholangitis and cholecystitis [4], the therapeutic strategy for the diagnosis and treatment of acute cholangitis and cholecystitis, based on accumulated evidence, is widely accepted. However, the therapeutic strategy for CBD stones differs among medical institutions and is controversial. According to a survey distributed by the Japan Society for Endoscopic Surgery, the use of a combination treatment of performing cholecystectomy (laparoscopic or open) after endoscopic treatment has been gradually increasing [2]. In the present case, the surgical procedure was performed according to the 2013 Tokyo Guidelines because endoscopic treatment was unsuccessful due to the Vater papilla opening in the third portion of the duodenum and the presence of a peripapillary duodenal diverticulum.

The rendezvous technique was originally one of many options to treat cholecystocholedocholithiasis. The rendezvous technique is a combined procedure in which the gallbladder is removed laparoscopically while a CBD stone is simultaneously cleared endoscopically by a selective CBD cannulation that is facilitated by the laparoscopic placement of a guide wire, through the cystic duct, into the duodenum.

Cavina et al. [5] reported for the first time in 1998 that the rendezvous technique in combination with LC was an efficacious method to treat choledocholithiasis, and they reported that there was no difference between the rendezvous technique and laparotomy or a combination of LC and EST in terms of the removal rates of CBD stones and the incidence of complications [5, 6]. Some reports have also indicated that the incidence of complications such as acute pancreatitis and bleeding in the rendezvous technique is lower than in the two-step approach (EST + LC), and that the rendezvous technique is significantly useful in shortening hospital stay and in reducing medical costs [7-9].

However, the rendezvous technique has not been used very much in Japan. There are two reasons. On the one hand, an extra-large operating room and numerous specialists are required to perform the rendezvous technique, and the various types of endoscopic devices required for the rendezvous technique are not covered by insurance in Japan. On the other hand, although laparoscopic CBD exploration enables earlier discharge than the two-step procedure, laparoscopic CBD exploration has some disadvantages linked to the incidence of complications such as bile leakage and postoperative biliary stricture [10-14]. The two-step procedure is thus widely accepted in Japan.

In the present case, since the patient was diagnosed as having mild acute cholangitis with a CBD stone and endoscopic biliary drainage was difficult due to the Vater papilla opening in the third portion of the duodenum and the peripapillary duodenal diverticulum, a surgical procedure was considered. However, since the diameter of the CBD was rather small, we suspected that if laparoscopic CBD exploration was performed, postoperative bile leakage and biliary stricture might occur $[7,15,16]$. Since transcystic lithotomy presents the possibility of recurrence of bile duct stones $[17,18]$, it was not selected. The rendezvous technique in combination with EST during LC was therefore selected.

Although the rendezvous technique is considered to be an efficacious and well-established method for treating choledocholithiasis, the organizational, logistic and technical problems that accompany this technique have not facilitated its use in Japan. When endoscopic treatment for choledocholithiasis is unsuccessful for any reason or when the CBD diameter is small and postoperative biliary stricture is suspected, the rendezvous technique can be a sufficiently effective method for choledocholithiasis. We were able to treat the present patient successfully and safely by using the rendezvous technique in combination with EST during LC. We note that the rendezvous technique is an attractive al- 
Tanaka et al.: Endoscopic Sphincterotomy Using the Rendezvous Technique for Choledocholithiasis during Laparoscopic Cholecystectomy: A Case Report

ternative for patients with any issues which make it difficult to perform preoperative ERCP or laparoscopic CBD exploration, as in this case.

\section{Disclosure Statement}

The authors have no conflicts of interest or funding to disclose.

\section{References}

1 Fitzgibbons RJ Jr, Gardner GC: Laparoscopic surgery and the common bile duct. World J Surg 2001;25: $1317-1324$.

2 Japan Society for Endoscopic Surgery: 11th nationwide survey of endoscopic surgery in Japan. J Jpn Soc Endosc Surg 2012;17:576-590.

3 Enochsson L, Lindberg B, Swahn F, et al: Intraoperative endoscopic retrograde cholangiopancreatography (ERCP) to remove common bile duct stones during routine laparoscopic cholecystectomy does not prolong hospitalization: a 2-year experience. Surg Endosc 2004;18:367-371.

-4 Takada T, Strasberg SM, Solomkin JS, et al; Tokyo Guidelines Revision Committee: TG13: Updated Tokyo Guidelines for the management of acute cholangitis and cholecystitis. J Hepatobiliary Pancreat Sci 2013;20: $1-7$.

5 Cavina E, Franceschi M, Sidoti F, et al: Laparo-endoscopic 'rendezvous': a new technique in the choledocholithiasis treatment. Hepatogastroenterology 1998;45:1430-1435.

6 La Greca G, Barbagallo F, Di Blasi M, et al: Rendezvous technique versus endoscopic retrograde cholangiopancreatography to treat bile duct stones reduces endoscopic time and pancreatic damage. J Laparoendosc Adv Surg Tech A 2007;17:167-171.

7 Morino M, Baracchi F, Migilietta C, et al: Preoperative endoscopic sphincterotomy versus laparoendoscopic rendezvous in patients with gallbladder and bile duct stones. Ann Surg 2006;244:889-893.

-8 Manes G, Baratti C, Ardizzone S, et al: Over-the-catheter precut to gain access to the biliary duct during ERCP rendezvous. Surg Endosc 2008;22:2323-2325.

-9 Tekin A, Ogetman Z, Altunel E, et al: Laparoendoscopic 'rendezvous' versus laparoscopic antegrade sphincterotomy for choledocholithiasis. Surgery 2008;144:442-447.

10 Cetta F: Do surgical and endoscopic sphincterotomy prevent or facilitate recurrent common duct stone formation? Arch Surg 1993;128:329-336.

11 Cuschieri A, Croce E, Faggioni A, et al: EAES ductal stone study. Preliminary findings of multi-center prospective randomized trial comparing two-stage vs single-stage management. Surg Endosc 1996;10: 1130-1135.

12 Berthou JC, Dron B, Charbonneau P, et al: Evaluation of laparoscopic treatment of common bile duct stones in a prospective series of 505 patients: indications and results. Surg Endosc 2007;21:1970-1974.

13 Rogers SJ, Cello JP, Horn JK, et al: Prospective randomized trial of LC+LCBDE versus ERCP/S+LC for common bile duct stone disease. Arch Surg 2010;145:28-33.

-14 Bansal VK, Misra MC, Rajan K, et al: Single-stage laparoscopic common bile duct exploration and cholecystectomy versus two-stage endoscopic stone extraction followed by laparoscopic cholecystectomy for patients with concomitant gallbladder stones and common bile duct stones: a randomized controlled trial. Surg Endosc 2014;28:875-885.

15 Croce E, Golia M, Azzola M, et al: Laparoscopic choledochotomy with primary closure. Follow-up (5-44 months) of 31 patients. Surg Endosc 1996;10:1064-1068.

-16 Lokesh HM, Pottakkat B, Prakash A, et al: Risk factors for development of biliary stricture in patients presenting with bile leak after cholecystectomy. Gut Liver 2013;7:352-356.

17 Stromberg C, Nilsson M, Leijonmarck CE: Stone clearance and risk factors for failure in laparoscopic transcystic exploration of the common bile duct. Surg Endosc 2008;22:1194-1199.

18 Bove A, Bongarzoni G, Palone G, et al: Why is there recurrence after transcystic laparoscopic bile duct clearance? Risk factor analysis. Surg Endosc 2009;23:1470-1475. 


\begin{tabular}{l|l}
\hline Case Rep Gastroenterol 2014;8:245-250 \\
\hline DOI: 10.1159/000367594 & $\begin{array}{l}\text { @ 2014 S. Karger AG, Basel } \\
\text { www.karger.com/crg }\end{array}$ \\
\hline
\end{tabular}

Tanaka et al.: Endoscopic Sphincterotomy Using the Rendezvous Technique for Choledocholithiasis during Laparoscopic Cholecystectomy: A Case Report
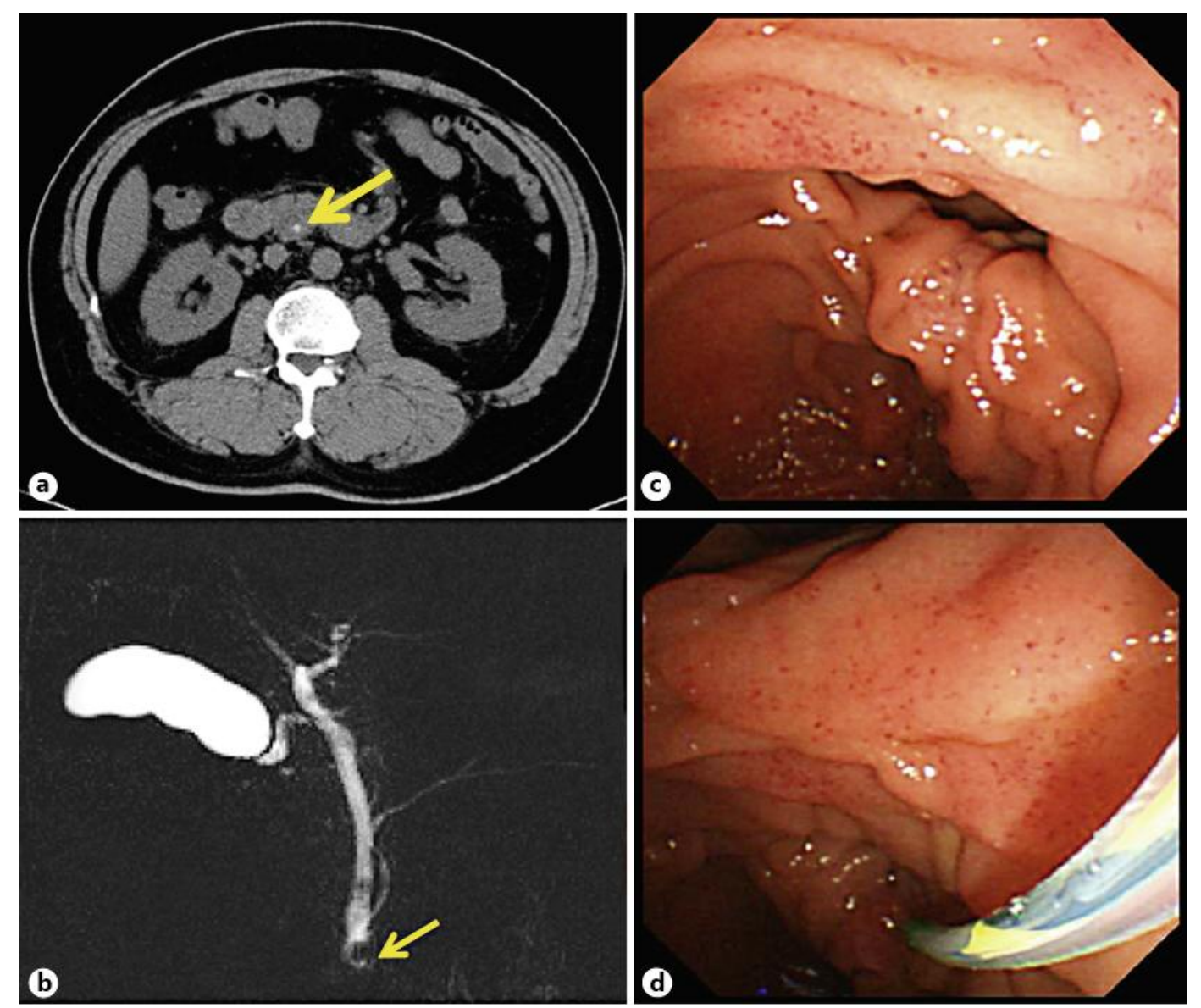

Fig. 1. Various imaging findings. a Abdominal computed tomography scan revealed that there was a CBD stone (arrow) and no findings suggestive of cholecystitis. b MRCP revealed a defect suggesting a CBD stone (arrow) and narrowing of the CBD (diameter $5 \mathrm{~mm}$ ). c ERCP revealed that there was a peripapillary duodenal diverticulum. $\mathbf{d}$ The peripapillary duodenal diverticulum made it impossible to insert the cannulation tube into the CBD. 


\begin{tabular}{l|l}
\hline Case Rep Gastroenterol 2014;8:245-250 \\
\hline DOI: 10.1159/000367594 & $\begin{array}{l}\text { @ 2014 S. Karger AG, Basel } \\
\text { www.karger.com/crg }\end{array}$ \\
\hline
\end{tabular}

Tanaka et al.: Endoscopic Sphincterotomy Using the Rendezvous Technique for Choledocholithiasis during Laparoscopic Cholecystectomy: A Case Report
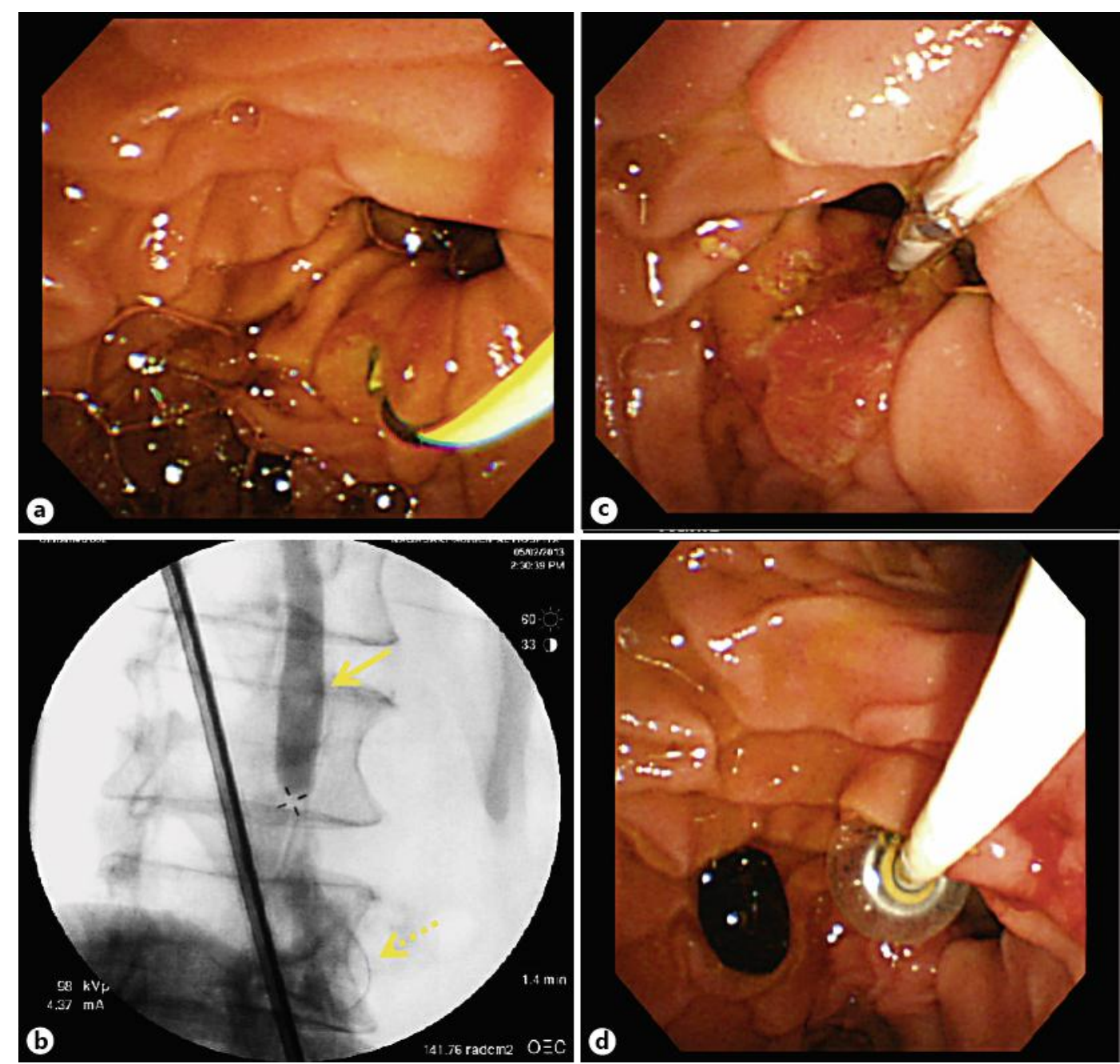

Fig. 2. EST during LC. a The guide wire was sent through the contrast tube to the duodenum through the papilla of Vater from the CBD. $\mathbf{b}$ X-ray also revealed that the guide wire was sent to the duodenum through the papilla of Vater (dashed arrow) and that there was a defect suggesting a stone (arrow). c The cutting knife was inserted and an intraoperative EST was performed. $\mathbf{d}$ Stone removal with a balloon was performed. 